\title{
Lucio Russo: L'America dimenticata. I rapporti tra le civiltà e un errore di Tolomeo
}

Mondadori Università, Milan, 2013

\author{
Liliana Curcio
}

Published online: 18 July 2014

(C) Centro P.RI.ST.EM, Università Commerciale Luigi Bocconi 2014

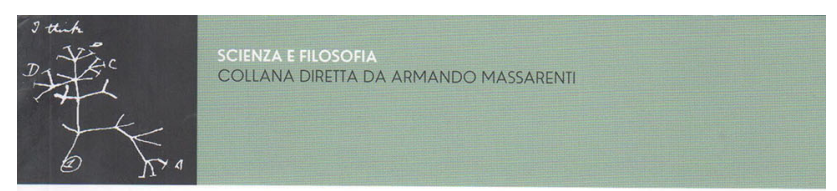

Lucio Russo

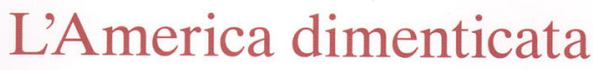

I rapporti tra le civiltà e un errore di Tolomeo

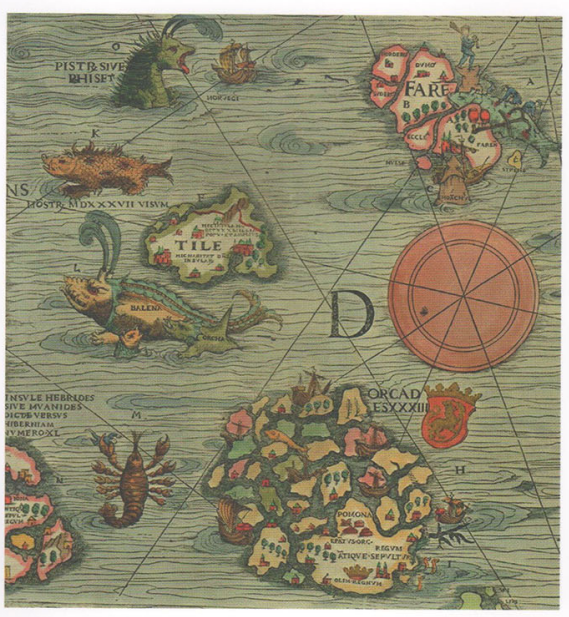

A. MONDADORI

Why did the celebrated Ptolemy (ca. 100-170 AD) commit a macroscopic error in measuring the dimensions of the earth, while Eratosthenes of Cyrene (ca. 275-195

L. Curcio $(\square)$

Largo Vulci 9, 20159 Milan, Italy

e-mail: liliana.curcio@unibocconi.it
BC), several centuries earlier, had calculated the same measurement with an almost exact result (with a deviation of less than $1 \%$ from the actual known dimension)?

This question, apparently of interest only to mathematicians, cartographers and geographers, becomes the point of departure for a fascinating investigation that gives rise to unexpected suppositions. In fact, Lucio Russo maintains the surprising hypothesis that the reason for Ptolemy's error lies in the collapse of knowledge that followed the Roman conquests. According to this theory, the broadening of territories conquered by Rome went hand-in-hand with a systematic destruction of the culture of all peoples conquered. One example is sufficient: the destruction of the Library of Alexandria and its inestimable patrimony of codices and volumes containing the entirety of human knowledge. In Russo's words:

This was not only the largest collection of books in existence in the world, but also the most important centre of editorial production, closely connected to the Musaeum of Alexandria, that is, to the principal public research institution of the ancient world, where scholars were lived at the expense of the state, devoting themselves to their own work without material worries (p. 90, our trans.).

The loss of knowledge was not limited to the scientific methodologies of mathematical geography and cartography, but also regarded the information about places and peoples, which often dated back to much earlier epochs. The recollections of past journeys also gradually disappeared, leaving in their wake tales that were legendary and unbelievable.

All of this is addressed in the book, starting with a mathematical key, a problem tied to mathematical geography: Ptolemy's error. With refined accuracy, Lucio Russo 
examines the routes taken and the inconsistencies of the results achieved by different scholars from Eratosthenes to Hipparchus all the way to Ptolemy.

The measurements of the scientists were made on the basis of tables previously compiled. Eratosthenes, who was director of the Library of Alexandria, used a procedure based on calculations within the framework of a mathematical model.

Ptolemy's error is due to a dilatation of the length in longitude, the consequence of which is a decrease in the size of the earth: 180,000 stadia for the length of the circumference instead of the 252,000 stadia obtained by Eratosthenes, which was much closer to the actual measurement. What is astonishing is that the dilatation of longitudes and the contraction of the dimensions of the earth are both aspects of the same error. While the latitudes of many localities are taken from direct sources and thus do not lead to the introduction of errors, the information available at the time regarding longitude required complex elaboration, and hence the error: the 'Fortunate Islands' that Ptolemy identified with the Canary Islands actually correspond to the Lesser Antilles (more precisely, the socalled Leeward Islands: the Americas!). The identification of the Fortunate Islands with the Canaries resulted in a chain of errors, leading Ptolemy to miscalculate the scale of longitudes and consequentially, to reduce the dimensions of the earth. From all of this it can be deduced that in the second century вС (and perhaps even earlier) the ships of the Mediterranean, and probably first of all those of the Carthaginians, not only reached the Caribbean but opened a route that was then used continuously. This would explain various of what appear to be historical inconsistencies, such as the representations of fruits that appear undoubtedly to be pineapples (a fruit native to the Antilles) in the works of artists and painters, and, in the opposite direction, the presence in the Americas of chickens, fowls of Eurasian origin, found by Christopher Columbus when he landed there.

Lucio Russo's hypothesis is fascinating, one that leads to deeper reflection and perhaps even to a revision of history books. It also leads us to consider the possibility that human societies arrive at the same results at the same time in very distant contexts, not by a kind of 'convergent evolution', but because they are mutually connected in a network of cultural exchanges and knowledge.

Translated from the Italian by Kim Williams.

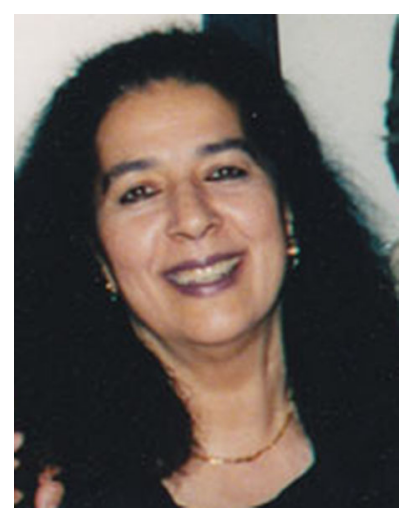

Liliana Curcio teaches mathematical analysis at the Politecnico di Milano. Formerly she taught mathematics and physics at the Istituto Statale d'Arte sperimentale (ISA) in Monza. She is a collaborator with the Centro Pristem, coordinating the courses of 'mathematical orientation' (Orientamatica) dedicated to high school students. She is a member of the Editorial Board of the Lettera Matemati$c a$, to which she has contributed various articles, with particular regard to the connections between mathematics, art and architecture. 